\title{
Skew Difference Algebras
}

\section{IVAN CHAJDA}

Department of Algebra and Geometry, Palacký University Olomouc, Tomkova 40, 77900 Olomouc, Czech Republic

e-mail : chajda@inf.upol.cz

ABSTRACT. We modify the definition of difference algebra given by J. Meng to obtain a structure which is a directoid with sectional switching involutions with respect to the given partial order. Moreover, we show that this is a representation of our skew difference algebras because every such directoid can be converted into a skew difference algebra.

The concept of a difference algebra was introduced by J. Meng [5] in the sake to axiomatize structures useful in non-classical propositional logics in general and their implicational reducts in particular. A number of examples of these algebras is presented in [5] and [6]. The original Meng's definition is as follows

Definition 1. A structure $(A ; *, \leq, 0)$ with a binary operation $*$, a nullary operation 0 and a binary relation $\leq$ is called a difference algebra if it satisfies the axioms

(D1) $(A ; \leq)$ is a poset;

(D2) $x \leq y$ implies $x * z \leq y * z$;

(D3) $(x * y) * z \leq(x * z) * y$;

(D4) $0 \leq x * x$;

(D5) $x \leq y$ if and only if $x * y \leq 0$.

However, this structure has a rather weak properties as a poset. To improve this, a concept of the so-called representable difference algebra was introduced in [2] in the sake to obtain a semilattice structure. However, it turns out that the concept of a representable difference algebra is too strong. It was shown recently [1] that this concept coincides with a commutative BCK-algebra. The reason is that some of axioms are rather strong and they cannot be used together. In particular, the axiom (D3) (usually called the exchange axiom) is too strong and we are wonder what will happen if it would be deleted. However, (D3) has several nice consequences which

Received April 27, 2007; revised September 1, 2009; accepted October 9, 2009.

2000 Mathematics Subject Classification: 06A06, 06A12, 03G25, 06F35.

Key words and phrases: difference algebra, skew difference algebra, sectional mapping, sectional switching involution, commutative directoid.

This work is supported by the Research and Development Council of the Czech Government via the project MSM6198959214. 
should be used for our considerations. Hence, we replace (D3) by one consequence of it and the list of axioms complete by the so-called quasi-commutativity axiom

$$
x *(x * y)=y *(y * x) .
$$

Then we are able to show that the modified difference algebra (called a skew difference algebra in the sequel) has a nice ordered structure (it is a commutative directoid) but it is not a semilattice and hence not a BCK-algebra. The definition is as follows:

Definition 2. By a skew difference algebra is meant a structure $\mathcal{S}=(S ; *, \leq, 0)$ where $*$ is a binary operation, 0 is a nullary operation and $\leq$ is a binary relation such that

(S1) $(S ; \leq)$ is a poset with the least element 0 ;

(S2) $x * x=0$;

(S3) $x \leq y$ if and only if $x * y=0$;

(S4) $(x * y) * x=0$;

(S5) $x *(x * y)=y *(y * x)$.

Hence, every skew difference algebra satisfying (D3) becomes a difference algebra with the least element 0 and satisfying (S5). Conversely, if $\mathcal{A}$ is a difference algebra such that 0 is its least element and satisfying (S5) then $\mathcal{A}$ is a skew difference algebra.

Further, we need several concepts which were already used for semilattices and lattices when characterizing MV-algebras and their modifications by the author and his collaborators, see e.g. [3].

Definition 3. Let $(S ; \leq, 0)$ be a poset with a least element 0 . For $a \in S$, the interval $[0, a]$ is called a section. A mapping $f_{a}:[0, a] \rightarrow[0, a]$ is called a sectional mapping. Instead of $f_{a}(x)$, we will write briefly $x^{a}$. A sectional mapping is a switching involution if $x^{a a}=x$ for each $x \in[0, a]$ and $a^{a}=0,0^{a}=a$. An ordered set $(S ; \leq, 0)$ is said to have sectional switching involutions if for each $a \in S$ there exists a sectional switching involution $x \mapsto x^{a}$ on $[0, a]$.

The following concept was introduced by J. Ježek and R. Quackenbush [4].

Definition 4. By a commutative directoid is meant a grupoid $(A ; \sqcap)$ satisfying the identities

(D1) $x \sqcap x=x$;

(D2) $x \sqcap y=y \sqcap x$;

(D3) $x \sqcap((x \sqcap y) \sqcap z)=(x \sqcap y) \sqcap z$. 
By a commutative directoid with $\mathbf{0}$ is meant a directoid $(A ; \sqcap)$ where $0 \in A$ and satisfying

(D4) $x \sqcap 0=0$.

The following facts can be taken from [4]:

(I) Let $(A ; \sqcap)$ be a commutative directoid. Define $\leq$ by the rule

$$
x \leq y \quad \text { if and only if } \quad x \sqcap y=x .
$$

Then $(A ; \leq)$ is a poset. If $(A ; \sqcap)$ is a commutative directoid with 0 then $0 \leq x$ for each $x \in A$.

(II) Let $(A ; \leq)$ be an arbitrary ordered set with a least element 0 . Denote by $L(x, y)=\{a \in A ; a \leq x$ and $a \leq y\}$ the set of common lower bounds of $x, y$. Of course, $L(x, y) \neq \emptyset$ since $0 \in L(x, y)$. Let $\varphi$ be an arbitrary mapping $\varphi: A \times A \rightarrow A$ satisfying the following

(a) $\varphi(x, y) \in L(x, y)$ and $\varphi(x, y)=\varphi(y, x)$;

(b) if $x \leq y$ then $\varphi(x, y)=x$.

Taking $x \sqcap y=\varphi(x, y)$, the derived structure $(A ; \sqcap)$ is a commutative directoid with 0 .

The following lemma will be useful for our computations.

Lemma 1. Let $\mathcal{S}=(S ; *, \leq, 0)$ be a skew difference algebra. Then

(i) $0 * x=0$;

(ii) $y *(y *(y * x))=y * x$;

(iii) $x * 0=x$.

Proof. (i) follows immediately from (S3) and the fact that $0 \leq x$ for each $x \in S$.

Prove (iii): Applying (S5) and (S2), we get

$$
x *(x * 0)=0 *(0 * x)=0 * 0=0
$$

due to (i). Hence, $x \leq x * 0$. However, (S4) yields $(x * 0) * x=0$ whence $x * 0 \leq x$. Thus $x * 0=x$.

Prove (ii): By (S5), (S4) and (iii) we infer

$$
y *(y *(y * x))=(y * x) *((y * x) * y)=(y * x) * 0=y * x .
$$

Theorem 1. Let $\mathcal{S}=(S ; *, \leq, 0)$ be a skew difference algebra. Define $x \sqcap y=$ $x *(x * y)$ as a term operation. Then $(S ; \sqcap)$ is a commutative directoid with 0 . 
Proof. By (S4) we get $(x *(x * y)) * x=0$ thus $x *(x * y) \leq x$. Using (S5), we conclude $x *(x * y)=y *(y * x) \leq y$, hence $x *(x * y) \in L(x, y)$. Suppose now $x \leq y$. By (S3) we have $x * y=0$ and, using (iii) of Lemma 1 , we compute

$$
x *(x * y)=x * 0=x .
$$

Hence, the mapping $\varphi(x, y)=x *(x * y)$ satisfies the afore mentioned conditions (a), (b) of (II) thus $(S ; \sqcap)$ for $x \sqcap y=x *(x * y)$ is a commutative directoid. Moreover, $x \sqcap 0=x *(x * 0)=x * x=0$ thus this directoid is with 0 .

In the sequel, the commutative directoid $(S ; \sqcap)$ reached in Theorem 1 will be referred as the induced directoid of $\mathcal{S}=(S ; *, \leq, 0)$.

Lemma 2. Let $\mathcal{S}=(S ; *, \leq, 0)$ be a skew difference algebra and $(S ; \sqcap)$ the induced directoid. Then the induced order of $(S ; \sqcap)$ coincides with $\leq$.

Proof. Assume $x \leq y$. Then $x * y=0$ and hence $x \sqcap y=x *(x * y)=x * 0=x$. Conversely, let $x \sqcap y=x$. Then $x *(x * y)=x$ and hence (by (S2) and (ii) of Lemma 1) $x * y=x *(x *(x * y))=x * x=0$ whence $x \leq y$.

Theorem 2. Let $\mathcal{S}=(S ; *, \leq, 0)$ be a skew difference algebra. For $a \in S$ and every $x \in[0, a]$ define $x^{a}=a * x$. Then the mapping $x \mapsto x^{a}$ is a sectional switching involution on the section $[0, a]$ for each $a \in S$.

Proof. Let $x \in[0, a]$. Then $x \leq a$. Due to (S3) we have $x^{a} * a=(a * x) * a=0$ thus $x^{a} \leq a$, i.e. the mapping $x \mapsto x^{a}$ is a sectional mapping on the section $[0, a]$. Further, using the induced directoid, we obtain $x^{a a}=a *(a * x)=a \sqcap x=x$ and $a^{a}=a * a=0,0^{a}=a * 0=a$, i.e. it is a switching involution on $[0, a]$.

Corollary. Let $\mathcal{S}=(S ; *, \leq, 0)$ be a skew difference algebra. Define $x \sqcap y=x *(x * y)$ and for $x \leq a, x^{a}=a * x$. Then $(S ; \sqcap)$ is a commutative directoid with 0 and with sectional switching involutions.

Our next goal is to prove the converse.

Theorem 3. Let $(S ; \sqcap)$ be a commutative directoid with 0 and with sectional switching involutions. Define $x * y=(x \sqcap y)^{x}$. Let $\leq$ be the induced order of $(S ; \sqcap)$. Then $\mathcal{S}=(S ; *, \leq, 0)$ is a skew difference algebra.

Proof. At first, $x \sqcap y \leq x$ thus $x \sqcap y \in[0, x]$ and hence the operation $x * y=(x \sqcap y)^{x}$ is correctly defined. As already mentioned in $(\mathrm{I}),(S ; \leq)$ is a poset with the least element 0 . We have to prove (S2)-(S5).

(S2): $x * x=(x \sqcap x)^{x}=x^{x}=0$.

(S3): Assume $x \leq y$. Then $x \sqcap y=x$ and hence $x * y=(x \sqcap y)^{x}=x^{x}=0$. Conversely, if $x * y=0$, then $(x \sqcap y)^{x}=0$ and, since the mapping $z \mapsto z^{x}$ is an involution (and hence bijection) which is switching, we conclude $x \sqcap y=x$. Hence $x \leq y$. 
(S4): Since $(x \sqcap y)^{x} \in[0, x]$, we have $x \leq(x \sqcap y)^{x}$ and hence $(x \sqcap y)^{x} \sqcap x=(x \sqcap y)^{x}$. Therefore $(x * y) * x=\left((x \sqcap y)^{x} \sqcap x\right)^{(x \sqcap y)^{x}}=\left((x \sqcap y)^{x}\right)^{(x \sqcap y)^{x}}=0$.

(S5): Since the operation $\sqcap$ is commutative, we have $x *(x * y)=x \sqcap y=y \sqcap x=$ $y *(y * x)$.

Example 1. We can illustrate the procedure described in Theorem 3 by the following example. Let $A=\{0, a, b, c, d, 1\}$ and $(A ; \sqcap)$ be a commutative directoid as shown in Fig. 1.

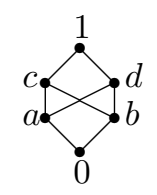

Fig. 1

Let the operation $\sqcap$ is defined as follows: if $x \wedge y$ exists then $x \sqcap y=x \wedge y$ and $c \sqcap d=a$. Sectional switching mappings are determined (in non-singleton sections) as follows.

Section $[0,1]: a^{1}=d, d^{1}=a, b^{1}=c, c^{1}=b, 0^{1}=1,1^{1}=0$.

Section $[0, c]: c^{c}=0,0^{c}=c, a^{c}=b, b^{c}=a$.

Section $[0, \mathrm{~d}]: d^{d}=0,0^{d}=d, a^{d}=a, b^{d}=b$.

Section $[0, \mathrm{a}]: a^{a}=0,0^{a}=a$.

Section $[0, \mathrm{~b}]: b^{b}=0,0^{b}=b$.

Then $(A ; \sqcap)$ is a commutative directoid with 0 and with sectional switching involutions. Define $x * y=(x \sqcap y)^{x}$. Then $*$ is given by the table

\begin{tabular}{c|cccccc|}
$*$ & 0 & $a$ & $b$ & $c$ & $d$ & 1 \\
\hline 0 & 0 & 0 & 0 & 0 & 0 & 0 \\
$a$ & $a$ & 0 & $a$ & 0 & 0 & 0 \\
$b$ & $b$ & $b$ & 0 & 0 & 0 & 0 \\
$c$ & $c$ & $b$ & $a$ & 0 & $b$ & 0 \\
$d$ & $d$ & $a$ & $b$ & $a$ & 0 & 0 \\
1 & 1 & $d$ & $c$ & $b$ & $a$ & 0 \\
\hline
\end{tabular}

One can easily check that $(A ; *, \leq, 0)$ is really a skew difference algebra.

In the remaining part, we can exhibit two very characteristic examples showing among other things that the directoid $(S ; \sqcap)$ need not be a semilattice and hence that skew difference algebras are far from commutative BCK-algebras.

Example 2. Consider the five element skew difference algebra whose table is 


\begin{tabular}{c|ccccc}
$*$ & 0 & $a$ & $b$ & $c$ & $d$ \\
\hline 0 & 0 & 0 & 0 & 0 & 0 \\
$a$ & $a$ & 0 & $a$ & 0 & 0 \\
$b$ & $b$ & $b$ & 0 & 0 & 0 \\
$c$ & $c$ & $b$ & $a$ & 0 & $b$ \\
$d$ & $d$ & $b$ & $a$ & $b$ & 0 \\
\hline
\end{tabular}

Then the induced ordered set is depicted in Fig. 2.

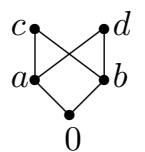

Fig. 2

In the induced directoid $(\{0, a, b, c, d\} ; \sqcap)$ we have $x \sqcap y=x \wedge y$ whenever $x \wedge y$ exists and $c \sqcap d=a$. The switching involutions in non-trivial (i.e. with more than two element) sections are as follows:

$$
\begin{aligned}
& {[0, \mathrm{c}]: \quad 0^{c}=c, c^{c}=0, a^{c}=b, b^{c}=a ;} \\
& {[0, \mathrm{~d}]: \quad 0^{d}=d, d^{d}=0, a^{d}=b, b^{d}=a .}
\end{aligned}
$$

Since there does not exist a greatest element of $L(c, d)=\{a, b, 0\},(S ; \sqcap)$, is not $\wedge$-semilattice and hence $(\{0, a, b, c, d\}, *, 0)$ is not a BCK-algebra.

Example 3. Consider the four element skew difference algebra whose table is

\begin{tabular}{c|cccc}
$*$ & 0 & $a$ & $b$ & $c$ \\
\hline 0 & 0 & 0 & 0 & 0 \\
$a$ & $a$ & 0 & 0 & 0 \\
$b$ & $b$ & $a$ & 0 & $b$ \\
$c$ & $c$ & $a$ & $c$ & 0 \\
\hline
\end{tabular}

The induced order is visualized in Fig. 3.

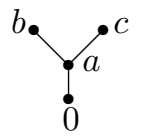

Fig. 3

The switching involutions in non-trivial sections are as follows:

$$
\begin{array}{ll}
{[0, \mathrm{~b}]:} & 0^{b}=b, b^{b}=0, a^{b}=a ; \\
{[0, \mathrm{c}]:} & 0^{c}=c, c^{c}=0, a^{c}=a .
\end{array}
$$

Although there can be defined a $\wedge$-semilattice structure on the poset $(\{0, a, b, c\} ; \leq)$, the induced directoid $(\{0, a, b, c\} ; \sqcap)$ is not a semilattice since

$$
b \sqcap c=b *(b * c)=b * b=0 .
$$




\section{References}

[1] Ahn S.S., Lee K.J., Remarks on "Representable difference algebras", Kyungpook Math. J., 46(2006), 433-436.

[2] Chajda I., Emanovský P., Representable difference algebras, Kyungpook Math. J., 44(2004), 335-342.

[3] Chajda I., Halaš R., Kühr J., Distributive lattices with sectionally antitone involutions, Acta Sci. Math. (Szeged), 71(2005), 19-33.

[4] Ježek J., Quackenbush R., Directoids: algebraic models of up-directed sets, Algebra Universalis, 27(1990), 49-69.

[5] Meng J., Difference algebras, Selected Papers on BCK- and BCI-algebras, 1(1992), 33-39.

[6] Roh E.H., Kim S.Y., Jun Y.B., Shim W.H., On difference algebras, Kyungpook Math. J., 43(2003), 407-414. 